\title{
Massive star formation in the W49 giant molecular cloud: Implications for the formation of massive star clusters
}

\author{
N. L. Homeier ${ }^{2,1}$ and J. Alves ${ }^{2}$ \\ ${ }^{1}$ Johns Hopkins University, Department of Physics and Astronomy, 21218 Baltimore, MD, USA \\ 2 European Southern Observatory, Karl-Schwarzschild Str. 2, 85748 Garching b. München, Germany \\ Received 15 March 2004 / Accepted 8 September 2004
}

\begin{abstract}
We present results from $J H K$ s imaging of the densest region of the W49 molecular cloud. In a recent paper (Alves \& Homeier 2003, ApJ, 589, L45), we reported the detection of (previously unknown) massive stellar clusters in the well-known giant radio HII region W49A, and here we continue our analysis. We use the extensive line-of-sight extinction to isolate a population of objects associated with W49A. We constrain the slope of the stellar luminosity function by constructing an extinction-limited luminosity function, and use this to obtain a mass function. We find no evidence for a top-heavy MF, and the slope of the derived mass function is $-1.6 \pm 0.3$. We identify candidate massive stars from our color-magnitude diagram, and we use these to estimate the current total stellar mass of 5-7 $\times 10^{4} M_{\odot}$ in the region of the W49 molecular cloud covered by our survey. Candidate ionizing stars for several ultra-compact HII regions are detected, with many having multipe candidate sources. On the global molecular cloud scale in W49, massive star formation apparently did not proceed in a single concentrated burst, but in small groups, or subclusters. This may be an essential physical description for star formation in what will later be termed a "massive star cluster".
\end{abstract}

Key words. ISM: H II regions - ISM: bubbles - Galaxy: open clusters and associations: individual: W49A - stars: formation Galaxy: disk - infrared: ISM

\section{Introduction}

The W49 Giant Molecular Cloud (GMC) is the most massive in the Galaxy outside the Galactic center; it extends over more than $50 \mathrm{pc}$ in diameter (Simon et al. 2001) with a mass of $\sim 10^{6} M_{\odot}$. Embedded within this cloud, W49A (Mezger et al. 1967; Shaver \& Goss 1970) is one of the brightest Galactic giant radio H II regions $\left(\sim 10^{7} L_{\odot}\right.$, Smith et al. 1978). As such, it has been used as a template for comparison with extragalactic "ultradense HII regions" (UD H II; Johnson \& Kobulnicky 2003), which appear to be massive star clusters in the process of assembly (Johnson et al. 2001; Vacca et al. 2002).

The W49A star-forming region lies in the Galactic plane $\left(l=43.17^{\circ}, b=+0.00^{\circ}\right)$ at a distance of $11.4 \pm 1.2 \mathrm{kpc}$ (Gwinn et al. 1992) and has 40 UC H II regions (e.g., De Pree et al. 1997, 2000; Smith et al. 2000) associated with a minimum of 40 central stars earlier than B3 (later than this, the star does not put out the necessary UV photons to ionize the surrounding gas, and it will not be detected as a UC H II region). About 12 of these radio sources are arranged in the well known Welch "ring" (Welch et al. 1987). A few other young Galactic clusters have a large number of massive stars, e.g., the Carina nebula (e.g. Walborn 1995; Rathborne et al. 2002), NGC 3603 (e.g. Moffat et al. 1994; Drissen et al. 1995; Eisenhauer et al. 1998; Brandl et al. 1999; Brandner et al. 2001; Moffat et al. 2002; Nürnberger \& Petr-Gotzens 2002; Sung \& Bessell 2004;
Stolte et al. 2004), Cygnus OB2 (e.g. Knödlseder 2000; Comerón et al. 2002; Hanson 2003), the Arches cluster (e.g. Serabyn et al. 1998; Blum et al. 2001b; Figer et al. 2002; Stolte et al. 2002), and Westerlund 1 (Clark \& Negueruela 2002), or are very young, e.g. NGC 3576 (Barbosa et al. 2003; Figuerêdo et al. 2002), W42 (Blum et al. 2000), and W31 (Kim \& Koo 2002; Blum et al. 2001a) but no other known region has a large number of massive stars in such a highly embedded and early evolutionary state. For this reason W49A is unique in our known Galaxy.

To uncover the embedded stellar population in W49A we performed a $5^{\prime} \times 5^{\prime}(16 \mathrm{pc} \times 16 \mathrm{pc})$, deep $J, H$, and $K$ s-band imaging survey centered on the densest region of the W49 GMC (Simon et al. 2001, see their Fig. 2). The initial results were presented in Alves \& Homeier (2003), where we reported the discovery of one massive and three smaller stellar clusters detected at NIR wavelengths. In this companion paper we present our photometric results, including the number of massive star candidates, objects with infrared excesses, and candidate ionizing sources of compact and ultracompact H II regions.

\section{Stages of massive star formation}

To better interpret what we observe in the W49A star-forming region, we will briefly mention a simplified version of the stages of massive star formation. The hot core phase is that 


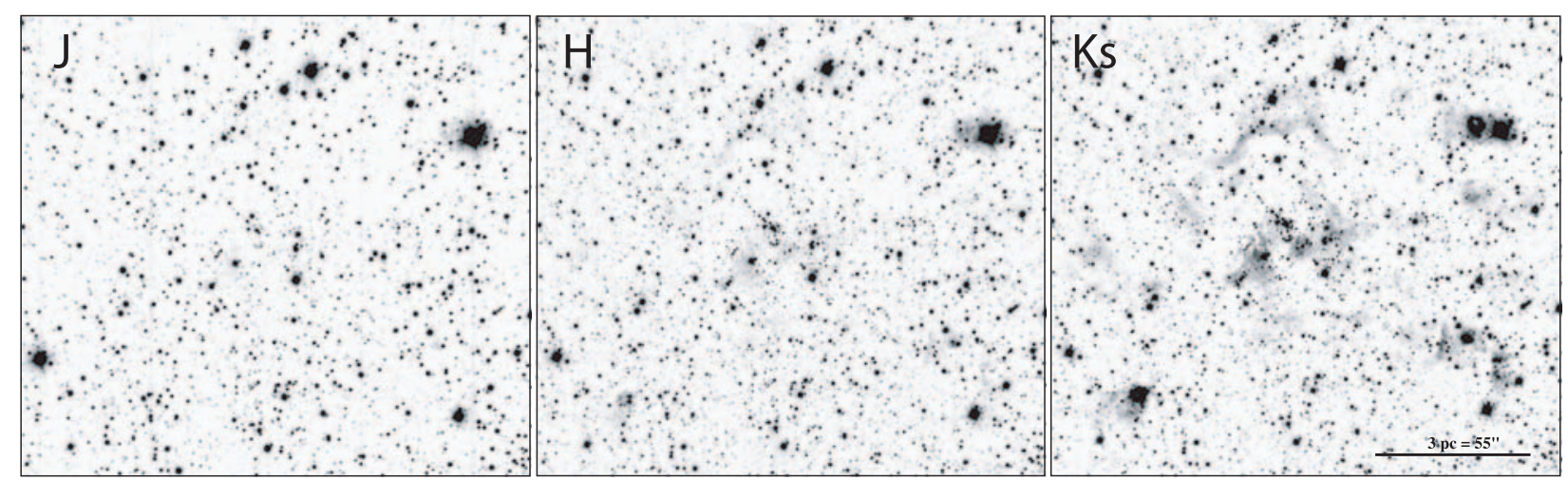

Fig. 1. $J, H$, and $K$ s images of the W49A region. The main star cluster begins to appear in the $H$ image, but is readily apparent in the $K$ s image, as are many diffuse nebular features. North is up and East to the left.

of a rapidly accreting, massive protostar. Although the protostar is emitting UV photons at this stage, the H II emission is "quenched" due to the high accretion rate (Walmsley 1995; Churchwell 2002). The next phase is the ultra-compact H II (UC H II) phase, and is the best studied one observationally. A UC H II region contains a central hydrogen-burning star which has ceased to accrete appreciably. The star's UV flux eats through its gas and dust cocoon and will eventually break out of the dense local medium to ionize surrounding more diffuse ISM. UC H II regions are radio-, far-IR-, and sometimes mid-IR-bright, but often undetectable at NIR wavelengths due to high local extinction. As the star disperses more of the surrounding gas and dust, the UC H II region becomes observable at shorter and shorter wavelengths, until the central object finally emerges as an unobscured massive star (see Churchwell 2002).

\section{Observations and data reduction}

The observations were taken on June 8, 2001, with the SOFI (Moorwood et al. 1998) near-infrared camera on the ESO's $3.5 \mathrm{~m}$ New Technology Telescope (NTT) on La Silla, Chile, during a spell of good weather and exceptional seeing ( $F W H M$ of the final combined images $\left.\sim 0.5-0.7^{\prime \prime}\right)$. SOFI employs a $1024 \times 1024$ Hawaii $\mathrm{HgCdTe}$ array, and the observations were taken with a plate scale of $0.288^{\prime \prime} /$ pixel. A set of 30 dithered images of $60 \mathrm{~s}$ each were taken in the $J, H$, and $K$ s filters. The images were combined with the DIMSUM ${ }^{1}$ package. The pixel scale of the final combined images is $0.15^{\prime \prime} /$ pixel. Standard stars 9136, 9157, and 9172 from the Persson catalog (Persson et al. 1998) were observed at the beginning, middle, and end of the night to obtain an airmass solution. Photometry was performed with the DAOPHOT package in IRAF $^{2}$. DAOFIND was used to detect sources above a threshold of 5 sigma. PSF models were constructed for each image using 5-7 isolated, bright stars, the tasks PSTSELECT, PSF, and ALLSTAR were run to

\footnotetext{
${ }^{1}$ DIMSUM is the Deep Infrared Mosaicking Software package developed by Peter Eisenhardt, Mark Dickinson, Adam Stanford, and John Ward, and is available via ftp from ftp://iraf.noao.edu/contrib/dimsumv3/

${ }^{2}$ IRAF is distributed by the National Optical Astronomy Observatories.
}

extract the final photometry, and aperture corrections were performed. $J$ and $H$ coordinates were transformed to $K$ s image coordinates using GEOMAP and GEOXYTRAN. Objects with errors larger than 0.15 mag and objects within 150 pixels of the image edges were excluded. Our final $J, H$, and $K$ s images are shown in Fig. 1. Our final samples contain 2255 and 7299, for the matched $J H K$ s and $H K$ s lists, respectively.

\subsection{Comparison with previous observations}

We compared our $K$ s photometry with the $K$ observations of Conti \& Blum (2002). From a sample of 493 stars in both data sets, we find an offset of $0.1 \mathrm{mag}$ between the $K$ and $K$ s magnitudes, with the SOFI photometry presented here being 0.1 mag fainter than the OSIRIS photometry. This offset can be accounted for by the different filter response curves and the highly reddened nature of the objects.

We performed tests with the SYNPHOT task CALCPHOT for the SOFI $K \mathrm{~s}$ and OSIRIS $K\left(K_{185}\right)$ filters. Using the Galactic extinction law of Clayton et al. (CCM) (1989) for $E(B-V)=10.85\left(A_{\mathrm{V}} \sim 30\right)$, SOFI $H-K \mathrm{~s}=1.561$ for a $30000 \mathrm{~K}$ blackbody, there is a $0.037-0.056$ mag difference for 30 000-3000 K blackbodies, with the SOFI photometry being fainter. With $E(B-V)=7.0\left(A_{\mathrm{V}} \sim 20\right)$, SOFI $H-K \mathrm{~s}=0.832$ for a $30000 \mathrm{~K}$ blackbody, there is a $0.014-0.029$ mag difference for 30000-3000 K blackbodies, again with the SOFI photometry being fainter. It would seem that approximately 0.04-0.09 mag are unaccounted for, however, the extinction laws available with SYNPHOT do not include the widely accepted Rieke \& Lebofsky (RL) (1985) Galactic extinction law, which we use throughout the rest of the paper. The slope of this extinction law also describes the slope of our color-color relation shown in Fig. 2.

For $A_{K}=1$, the CCM law gives $H-K=0.6$, while the RL law gives $H-K=0.57$. Thus there is a difference of 0.03-0.09 mag for $A_{K}=1-3$, typical of the stars in this region. Therefore, if the RL extinction law (and not the CCM law) accurately describes the extinction along the line of sight to W49A (which we have evidence for) then the $0.1 \mathrm{mag}$ offset between our SOFI $K$ s magnitudes and the $K$ magnitudes of Conti \& Blum (2002) should be due to the different filter response curves and the highly reddened nature of the stars. 


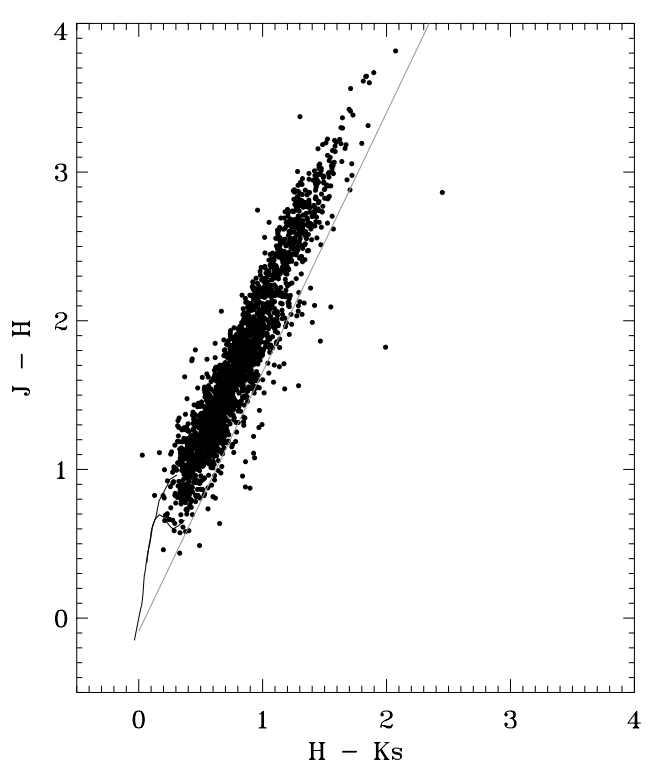

Fig. 2. Color-color diagram for our survey. The main sequence and giant tracks are overplotted, as is a light solid line indicating the reddening sequence for the bluest, hottest stars. A Rieke \& Lebofsky (1985) extinction law was used.

\subsection{Completeness limits}

The completeness limits were determined by adding 500-1500 fake stars to each image and extracting them in the same way in which the data analysis was performed. The fake stars were created with the psf image used with the ALLSTAR task, and input to the science images with ADDSTAR. We consider a star as recovered only if its recovered magnitude is within $0.15 \mathrm{mag}$ (our error cut) of the input magnitude. The $80 \%$ completeness limits for the $J, H$, and $K$ s filter images are 20.0,18.7, and 17.2 , respectively. The limits reflect the increasing importance of crowding in our images from $J$ to $K \mathrm{~s}$.

Because of crowding concerns, we also performed completeness tests on the central $500 \times 500$ pixels of our images. For the $H$ and $K$ s images, the $80 \%$ completeness limits were approximately $0.5 \mathrm{mag}$ brighter than the limits for the entire field. The $J$ limit was unaffected.

\section{Results}

\subsection{Luminosity functions}

Our images contain many stars along the line of sight, but we can use the reddening within the Galactic disk to our advantage. We attempt to identify a stellar population associated with the W49A region by first selecting objects with $H-K$ s colors red enough to be consistent with a distance of $11.4 \mathrm{kpc}$ along the Galactic plane. This can be calculated by assuming an exponential distribution of dust (as in Homeier et al. 2003) so that the extinction follows the form:

$a_{K}(R)=a_{K, 0} \mathrm{e}^{-\left(R-R_{0}\right) / \alpha_{R}}$, and

$R=\left(x^{2}+R_{0}^{2}-2 x R \cos l\right)^{1 / 2}$,



Fig. 3. The $K$-band luminosity function for all objects. The binsize is 0.3 mag. We show the $80 \%$ completeness limit as a dashed line.

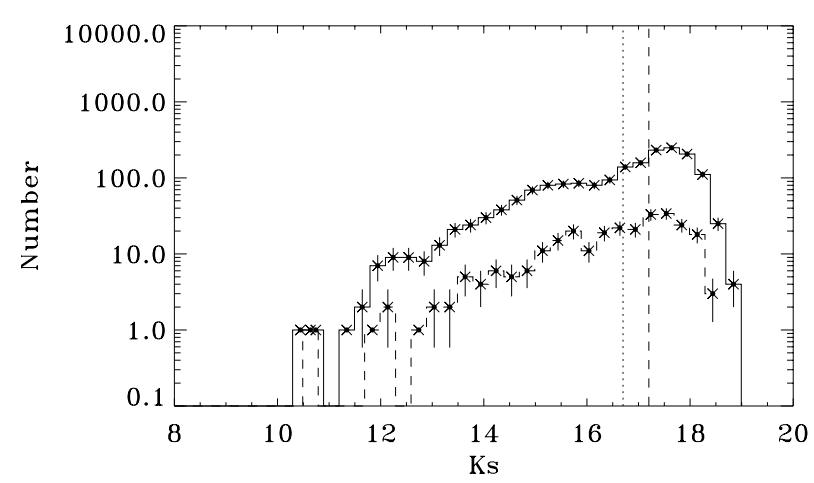

Fig. 4. The $K$-band luminosity function for objects with $H-K \geq 1.2$ which are likely to be associated with the W49A star-forming region (see text). The binsize is $0.3 \mathrm{mag}$. The dashed histogram indicates objects within $45^{\prime \prime}$ of our adopted center of Cluster 1 . We include the $80 \%$ completeness limit for the entire field as a dashed line, and for the inner $500 \times 500$ pixels as a dotted line.

where $x$ is $11.4 \pm 1.2 \mathrm{kpc}$ from Gwinn et al. (1992), $l=43$, $R_{0}=8 \mathrm{kpc}$, and $\alpha_{R}=3.0 \mathrm{kpc}$ (Kent et al. 1991). For these parameters we arrive at $A_{K}=2.1$ and $H-K=1.2$ for a Rieke \& Lebofsky (1985) reddening law.

In the remainder of the paper, we consider objects within $45^{\prime \prime}$ of $19 \mathrm{~d} 10 \mathrm{~m} 17.5 \mathrm{~s}, 09 \mathrm{deg} 06^{\prime} 21^{\prime \prime}$ (J2000) to be associated with Cluster 1, which corresponds to the arc of ionized emission to the North, and a physical distance of $2.5 \mathrm{pc}$.

In Fig. 3 we present the $K$-band luminosity function for all objects in our $H$ and $K$ sample, and in Fig. 4 we select only those objects with $H-K \geq 1.2$ as being located at or farther than the W49 molecular cloud as described above. The histogram for objects within $45^{\prime \prime}$ of Cluster 1 is plotted with a dashed line, and a solid line indicates all objects outside this region. The binsize is $0.3 \mathrm{mag}$, and the bin boundary at the faint magnitude limit was chosen to be the magnitude of the faintest star in each sample.

We would like an unbiased luminosity function for the stars associated with W49, so we select an extinction-limited sample of stars within $45^{\prime \prime}$ of our adopted center of Cluster 1 . We expect negligible background contamination near Cluster 1 due to the large optical depth of the W49 molecular cloud. The magnitude and color limits of our extinction-limited sample are shown in Fig. 5. The $H-K \mathrm{~s}=1.2$ limit is set by our best estimate of foreground extinction as previously described. 


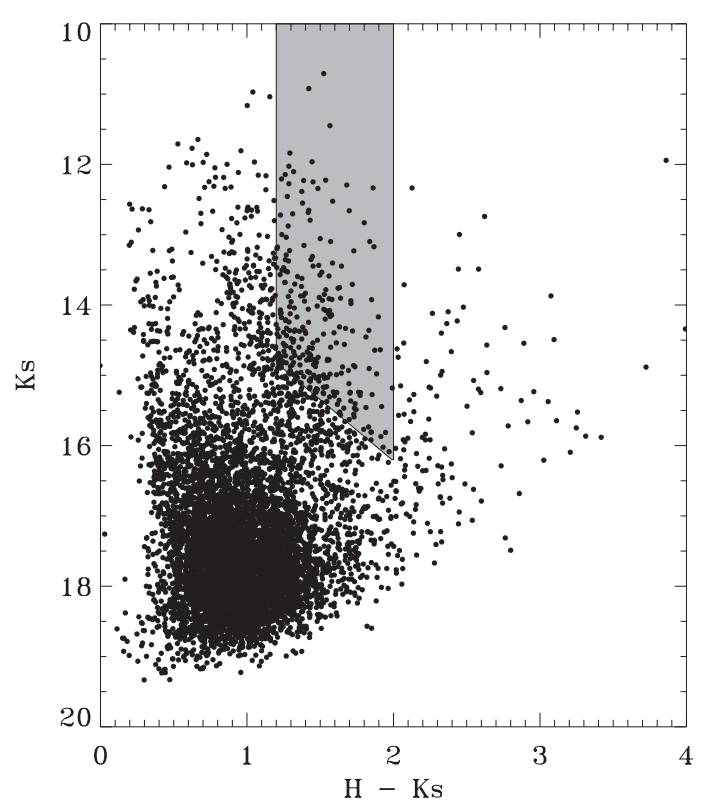

Fig. 5. $(H-K \mathrm{~s})$ Color-magnitude diagram showing the limits for an extinction-limited sample. We chose an $H-K=2.0$ limit to cover a reasonable range of reddening. For the entire field, our $80 \%$ completeness limits are $H=18.7$ and $K \mathrm{~s}=17.2$. However, crowding in the center of the image reduces this to $H=18.2, K \mathrm{~s}=16.7$ Thus at $K \mathrm{~s}=16.2, H-K=2$, we are above $80 \%$ completeness for everything brighter and bluer than these limits. This defines the bottom-right corner of the overplotted polygon. The slope of the bottom edge is determing by the extinction law, and the $H-K=1.2$ limit is described in Sect. 4.1.



Fig. 6. Extinction-limited $K$-band luminosity function for Cluster 1. The binsize is $0.5 \mathrm{mag}$.

Our $80 \%$ completeness limits for the entire field are at $H=$ 18.7 and $K \mathrm{~s}=17.2$. Thus at $K \mathrm{~s}=16.7, H-K \mathrm{~s}=2$, we are above $80 \%$ completeness for everything brighter and bluer than these limits. However, crowding in the center reduces these $80 \%$ completeness limits to $H=18.2$ and $K \mathrm{~s}=16.7$, and thus $K \mathrm{~s}=16.2, H-K \mathrm{~s}=2$ defines the bottom-right corner of the overplotted region. The slope of the bottom edge is determined by a Rieke \& Lebofsky extinction law (1985).

The extinction-limited KLF for Cluster 1 is shown in Fig. 6, and for everything in our field in Fig. 8. For these histograms, the bin boundary at the faint end was set at the faintest star in each sample. The error bars are $\sqrt{N}$, where $N$ is the number of stars in each bin.

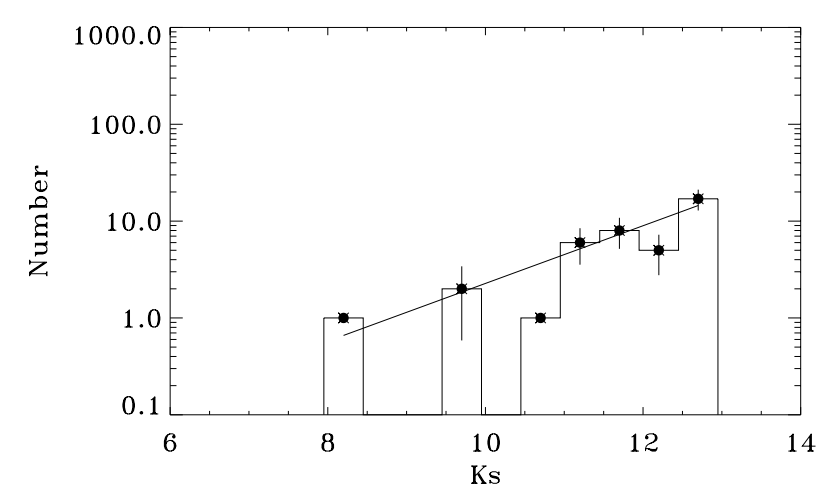

Fig. 7. Extinction-limited extinction-corrected $K$-band luminosity function for Cluster 1 . The binsize is $0.5 \mathrm{mag}$. The slope of the fitted line is $0.30 \pm 0.16$. We used a larger bin size to take into account extinction correction errors caused by intrinsic $H-K$ s colors.



Fig. 8. Extinction-limited $K$-band luminosity function for all objects. The binsize is $0.5 \mathrm{mag}$.

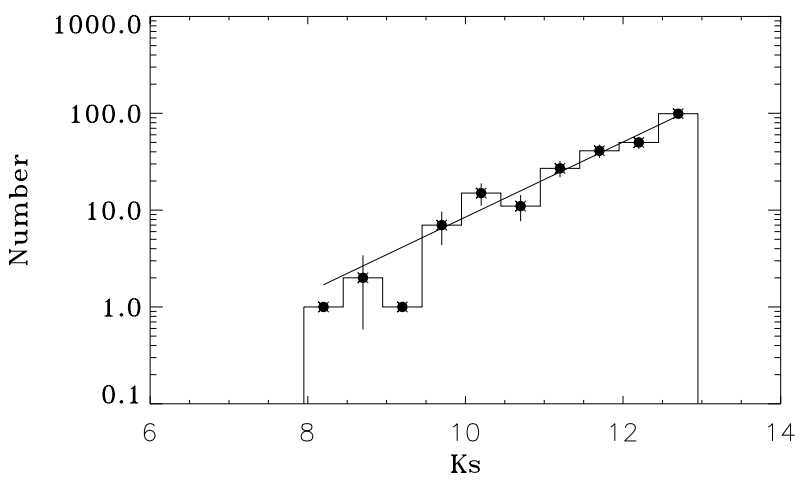

Fig. 9. Extinction-limited extinction-corrected $K$-band luminosity function for all objects. The binsize is $0.5 \mathrm{mag}$. The slope of the fitted line is $0.37 \pm 0.07$. We used a larger bin size to take into account extinction correction errors caused by intrinsic $H-K$ s colors.

Since both samples suffer from severe non-uniform extinction, we corrected for this effect assuming an intrinsic color of $H-K s=0.15$. This choice is based on the knowledge that all stars without hot dust are intrinsically nearly colorless in the near-infrared, with $H-K$ s ranging from 0.0 to 0.3 . We expect objects associated with the W49A starforming region to be early-type stars with intrinsic $H-K \mathrm{~s}$ near 0.0, whereas giant stars should have intrinsic $H-K$ s up to 0.3 . Thus we calculate extinction-corrected $K$ magnitudes 
as $K_{\text {corr }}=K_{\text {obs }}+((H-K \mathrm{~s}-0.15) / 0.57)$. Dereddening a star with an intrinsic color $H-K \mathrm{~s}=0$ to $H-K \mathrm{~s}=0.15$ will result in an inferred $K \mathrm{~s}$ magnitude which is 0.25 mag too faint, whereas a star with an intrinsic $H-K \mathrm{~s}=0.3$ will be $0.25 \mathrm{mag}$ too bright.

Our extinction-corrected extinction-limited KLFs for Cluster 1 and for all objects in our field are shown in Figs. 7 and 9. We use $0.5 \mathrm{mag}$ bins for these samples to take into account the uncertainty in the extinction correction. The bin boundary at the faint end was set to $K=12.95$, the faintest extinction-corrected magnitude allowed by our selection criteria. A linear least-squares fit was made, yielding a slope of $0.30 \pm 0.16$ for Cluster 1 and $0.37 \pm 0.07$ for the entire field.

\subsection{Mass function}

Any photometric mass function relies on a magnitude-mass relation, which has its source in a luminosity-mass relation. We take the relationship between initial mass and absolute $K$ magnitude from the $4 \times 10^{5} \mathrm{yr}$ isochrones of Lejeune \& Schaerer (2001) with enhanced mass loss rates. We can then construct a mass function by converting our extinction-limited extinctioncorrected $K$ s luminosity function for our entire field to a mass function by transforming each magnitude bin to a mass bin. We can also convert magnitudes for individual stars into masses, then bin these masses to arrive at a mass histogram. The mass functions derived in these two ways are shown in Fig. 10. We extrapolated the magnitude-mass relation to infer masses for the most luminous stars, which are more luminous than the $120 M_{\odot}$ models. Errors of $10-20 \%$ in the mass estimates are expected simply from the uncertainty in the distance.

Our slopes are derived from linear least-squares fits weighted with the errors derived from Poisson statistics $(\sqrt{N})$. The mass function slopes yielded by the two methods, $-1.60 \pm 0.28$ and $-1.64 \pm 0.28$, are in excellent agreement The error in each slope measurement is large and there are many sources of uncertainty, but we can conclude that we do not find evidence for a top-heavy IMF. If we use the $1 \mathrm{Myr}$ isochrones, our measured slopes are $-1.70 \pm 0.30$ and $-1.57 \pm 0.31$, within the $1 \sigma$ uncertainties.

The slope of our mass function and the number of stars in the sample indicate that we should have at least one $M>$ $200 M_{\odot}$ in our extinction-limited sample. Our $J$ image does not go deep enough for us to securely identify extremely massive candidates. There are several luminous objects at $K$ s for which we lack $J$ magnitudes, and we are therefore unable to quantify the contribution to the $K$ s magnitude from hot dust.

\subsection{Candidate massive stars}

To estimate the number of stars with masses $\geq 20 M_{\odot}$ associated with the W49A region, we will assume intrinsic colors of $H-K=0$ and calculate the unobscured apparent $K$ magnitude as $K_{\mathrm{obs}}+(0.57 / H-K)$. As in the previous section, we use the relation between mass and absolute $K$ magnitude from the Lejeune \& Schaerer (2001) models at $4 \times 10^{5} \mathrm{yr}$ for solar metallicity and enhanced mass loss. Assuming an age from
$3 \times 10^{5}$ yr to 2 Myr has a negligible effect on our overall result.

Figure 11 shows our CMD with the position of a $4 \times$ $10^{5}$ yr $20 M_{\odot}$ star, and a reddening line indicating $A_{K}=5$. All stars above this line with $H-K \geq 1.2$ are identified as candidate massive stars. We will use this sample later to estimate the total stellar mass in the region.

\subsection{Contamination}

There is no robust way to measure the background in such a region, as the cluster is embedded in a molecular cloud, which means the extinction is non-uniform across the field. However, one likely contaminant is disk giant stars. Absolute $K$ s magnitudes for the brightest disk giant stars should be $\sim-4.7$ (Sparke \& Gallagher 2000), which is equivalent to an apparent magnitude of $\sim 10.1$ at a distance of $12 \mathrm{kpc}$ (neglecting extinction), just farther than the W49A region. Assuming $A_{K}=2-2.5 \mathrm{mag}$, they would have apparent magnitudes of $\sim 12.1-12.6$. If we select stars with $H-K \mathrm{~s}>1.2$ and $K \mathrm{~s}$ in this magnitude range, we find that they are not uniformly distributed over our field, but fall preferentially on the northern half. This is consistent with their identification as background giant stars, as the W49 molecular cloud is less dense as one goes from the center to the northern edge of the image. From the non-uniform distribution of reddened sources in our field, which we identify as background giants, we estimate that they contribute 20-30 stars to our total. Another source of contamination in our census of massive stars is a possible population of stars which are undetected at $J$ with $K$ s excesses, which could make some less massive stars appear as more massive stars.

\subsection{Objects with infrared excesses}

Our $J-H$ vs. $H-K$ s color-color diagram is shown in Fig. 2, with the main sequence and giant tracks overplotted as solid and dotted lines (Bessell \& Brett 1988). The reddening boundary for the hottest stars is plotted as a dashed line. We can see that several stars fall to the right of this line, which would indicate an $H-K$ s color which is affected not only by extinction, but also a $K$ s excess due to hot dust. However, most of these are not extincted enough to be part of the W49 region, and also fall near the edges of our images. These could be photometric outliers, or true Ks excess objects along the line of sight; most probably, the population is a combination of the two. Only two stars with strong $K$ s excesses are likely to be part of the W49A region. Both of these are within $2 \mathrm{pc}$ of the projected center of Cluster 1. One is faint and appears to have an unresolved companion at $\mathrm{K}$, suggesting the result could be due to a deblending error. The other was identified as star No. 2 by Conti \& Blum (2002). This object has an excess of approximately 0.7 mag and thus from its corrected color and magnitude appears to be a star with a mass of 20-25 $M_{\odot}$. It is located between the projected center of Cluster 1 and the ring of ultracompact HII regions. This star is an obvious candidate for follow-up observations looking for evidence that the hot dust which surrounds the star is in the shape of a remnant accretion disk. 

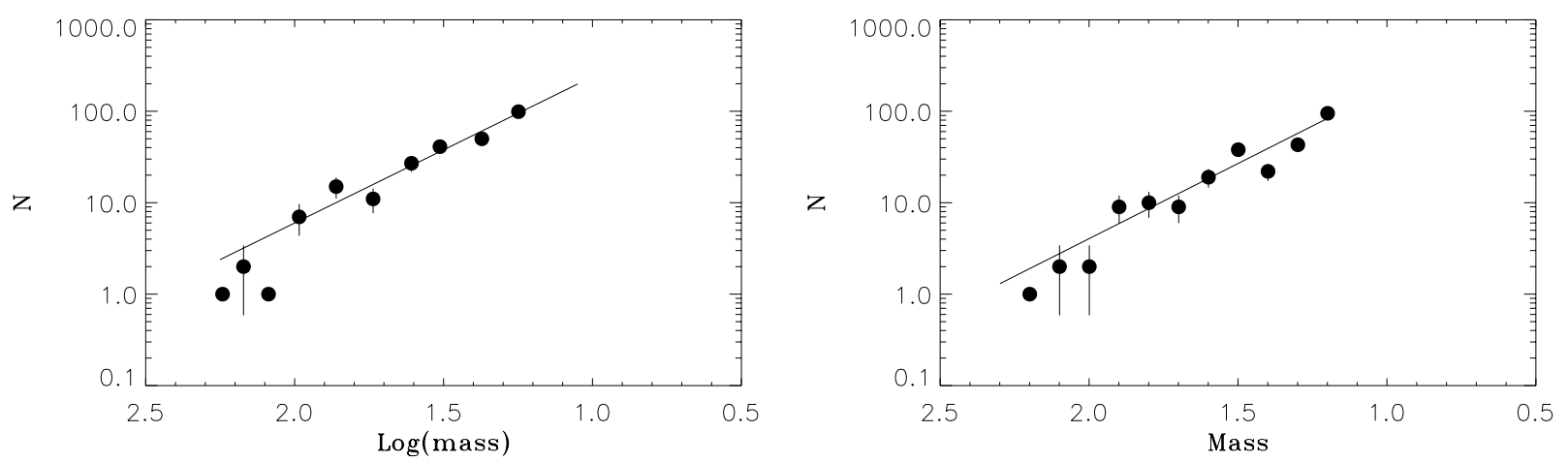

Fig. 10. Mass functions from the extinction-limited extinction-corrected sample shown in Fig. 9. In the left panel we transformed the $K$ s magnitudes of the luminosity function into mass. The mass bin sizes thus depend on the mass-magnitude relationship. The fitted slope is $-1.60 \pm 0.28$. In the right panel, masses were derived based on the $K$ s magnitudes for individual stars, and the $\log (M)$ bins are 0.1 . The fitted slope is $-1.64 \pm 0.28$. We do not find evidence for a top-heavy IMF.



Fig. 11. $(H-K)$ Color-magnitude diagram showing the limits for identifying candidate massive stars. The solid line represents the reddening line for a $4 \times 10^{5}$ yr $20 M_{\odot}$ star (Lejeune \& Schaerer 2001), from $A_{K}=0$ to $A_{K}=5$. The dotted line represents the $H-K=1.2$ limit described in Sect. 4.1.

\subsection{Candidate ionizing sources of compact and ultracompact $H I I$ regions}

Conti \& Blum (2002) detected two UCH II regions in their $H$ and $K$ images, radio sources " $F$ " and "J2". We also detect these sources, and in Table 1 we list candidate ionizing sources for the compact and ultracompact $\mathrm{H}$ II regions CC, F, J2, R and Q, S, and W49 South (names from De Pree et al. 1997). For regions with multiple detections, we have selected only objects with inferred $M>15 M_{\odot}$. We list inferred masses in Col. 9, and we note that with the relation we are using, five objects have inferred masses greater than $120 M_{\odot}$. This corresponds to an absolute $K$ magnitude brighter than -6.2 . For the stars without $J$ magnitudes, they could have infrared excesses which push their $K$ s magnitudes above this.

This is almost certainly the case for object F. Unpublished spectra indicate that it has a spectrum marked only by lines of He I at $2.06 \mu \mathrm{m}, \mathrm{Br} \gamma$, and anomalous features at $2.08 \mu \mathrm{m}$ in emission and 2.10 in absorption (P. Conti \& P. Crowther, private communication). The important point is that no photospheric lines are seen. We can put an upper limit of 20 on its $J$ magnitude, for a minimum $J-H$ color of 4.2 , and a maximum $K$ s excess of $\sim 1.5 \mathrm{mag}$.

One of the objects with inferred $M>120 M_{\odot}$ has $J-H$ and $H-K$ s colors that indicate it does not have a $K$ s excess. It has an inferred absolute $K$ magnitude of -7.14 , which is highly overluminous, even for a multiple of 3 objects. One possibility is that this object is slightly older than the surrounding stars. The stellar evolutionary models for high mass stars predict that a $120 M_{\odot}$ star will enter the supergiant phase at $1.7 \mathrm{Myr}$, and brighten by about $1.5 \mathrm{mag}$ in $K$. An age spread of $1 \mathrm{Myr}$ would explain this. Another less interesting possibility is that the magnitudes are simply off due to difficulty in correctly characterizing the surrounding nebular emission.

\section{Discussion}

\subsection{The W49A star cluster}

The virial mass of the W49 molecular cloud, $10^{6} M_{\odot}$, puts it among the most massive in our galaxy (Simon et al. 2001). Our NIR observations cover the densest regions of this cloud, over a physical distance of $15 \mathrm{pc}$. There are "fuzzy" patches in our $K$ s image from nebular emission, and these extend to the Eastern, Western, and Southern edges of our field, indicating that we have not fully sampled the star formation activity in the W49A cloud. There is also a peak in both the molecular gas density (Simon et al. 2001) and the radio emission (Brogan \& Troland 2001) to the Northeast of our field.

What we have uncovered is a previously undetected massive stellar cluster (Cluster 1), and stellar sources associated with UC H II regions. Cluster 1 and the "ring" of UC H II regions are separated by only 2 pc in projection, meaning that this differs from a "second generation" as seen in 30 Doradus (Walborn et al. 1999, 2002) and NGC 3603 (Blum et al. 2000; Nürnberger et al. 2002). In the case of W49A, when the $\mathrm{OB}$ stars powering the $\mathrm{UC} \mathrm{HII}$ regions emerge, the region encompassing both Cluster 1 and the Welch ring of UC HII 
Table 1. Candidate stars associated with HII regions.

\begin{tabular}{llllccccc}
\hline \hline Radio source & RA $(2000)$ & Dec $(2000)$ & $J$ & $H$ & $K$ s & $J-H$ & $H-K$ s & Inferred mass $\left(M_{\odot}\right)$ \\
\hline CC & $19: 10: 11.60$ & $9: 07: 06.5$ & $19.09 \pm 0.02$ & $15.78 \pm 0.02$ & $13.92 \pm 0.04$ & 3.31 & 1.86 & 56 \\
F & $19: 10: 13.42$ & $9: 06: 22.0$ & - & $15.80 \pm 0.01$ & $11.94 \pm 0.02$ & - & 3.86 & $>120 ?$ \\
J2 & $19: 10: 14.22$ & $9: 06: 27.4$ & - & $17.36 \pm 0.01$ & $15.45 \pm 0.02$ & - & 1.91 & 25 \\
O3 & $19: 10: 16.92$ & $9: 06: 10.9$ & $18.61 \pm 0.02$ & $15.54 \pm 0.02$ & $13.90 \pm 0.03$ & 3.07 & 1.64 & 46 \\
RQ & $19: 10: 10.76$ & $9: 05: 18.2$ & - & $19.18 \pm 0.03$ & $15.87 \pm 0.03$ & - & 3.31 & 78 \\
RQ & $19: 10: 10.93$ & $9: 05: 15.9$ & - & $17.33 \pm 0.02$ & $15.63 \pm 0.02$ & - & 1.70 & 18 \\
RQ & $19: 10: 10.96$ & $9: 05: 17.7$ & - & $18.57 \pm 0.03$ & $12.94 \pm 0.02$ & - & 5.64 & $>120 ?$ \\
RQ & $19: 10: 11.16$ & $9: 05: 11.6$ & $17.84 \pm 0.01$ & $15.51 \pm 0.02$ & $14.36 \pm 0.02$ & 2.34 & 1.14 & 21 \\
S & $19: 10: 11.66$ & $9: 05: 27.5$ & $16.48 \pm 0.02$ & $14.16 \pm 0.02$ & $13.02 \pm 0.04$ & 2.32 & 1.14 & 46 \\
S & $19: 10: 11.80$ & $9: 05: 27.1$ & - & $18.19 \pm 0.04$ & $15.23 \pm 0.05$ & - & 2.96 & 80 \\
S & $19: 10: 11.83$ & $9: 05: 28.3$ & - & $17.21 \pm 0.03$ & $14.57 \pm 0.04$ & - & 2.64 & 84 \\
S & $19: 10: 11.88$ & $9: 05: 28.2$ & - & $16.73 \pm 0.01$ & $14.40 \pm 0.02$ & - & 2.33 & 68 \\
South & $19: 10: 21.90$ & $9: 04: 57.1$ & - & $17.60 \pm 0.02$ & $14.96 \pm 0.04$ & - & 2.64 & 68 \\
South & $19: 10: 22.08$ & $9: 05: 00.0$ & - & $17.43 \pm 0.02$ & $14.54 \pm 0.03$ & - & 2.89 & 111 \\
South & $19: 10: 22.09$ & $9: 05: 01.5$ & - & $19.02 \pm 0.04$ & $16.29 \pm 0.02$ & - & 2.73 & 36 \\
South & $19: 10: 21.97$ & $9: 05: 04.2$ & - & $16.61 \pm 0.02$ & $14.54 \pm 0.03$ & - & 2.07 & 49 \\
South & $19: 10: 22.20$ & $9: 05: 04.2$ & - & $18.23 \pm 0.03$ & $16.19 \pm 0.02$ & - & 2.05 & 19 \\
South & $19: 10: 21.90$ & $9: 04: 58.1$ & - & $16.78 \pm 0.05$ & $14.74 \pm 0.04$ & - & 2.04 & 42 \\
South & $19: 10: 22.20$ & $9: 05: 01.5$ & - & $18.06 \pm 0.03$ & $15.84 \pm 0.04$ & - & 2.22 & 28 \\
South & $19: 10: 21.80$ & $9: 05: 03.3$ & - & $18.33 \pm 0.03$ & $14.34 \pm 0.05$ & - & 3.99 & $>120 ?$ \\
South & $19: 10: 21.88$ & $9: 05: 04.3$ & $20.07 \pm 0.04$ & $15.36 \pm 0.01$ & $12.74 \pm 0.03$ & 4.71 & 2.62 & $>120 ?$ \\
South & $19: 10: 21.75$ & $9: 05: 04.3$ & - & $18.61 \pm 0.03$ & $14.88 \pm 0.05$ & - & 3.73 & $>120 ?$ \\
South & $19: 10: 22.24$ & $9: 05: 00.3$ & - & $18.46 \pm 0.01$ & $16.29 \pm 0.03$ & - & 2.17 & 20 \\
South & $19: 10: 22.02$ & $9: 05: 00.3$ & - & $18.27 \pm 0.03$ & $16.11 \pm 0.05$ & - & 2.16 & 22 \\
South & $19: 10: 22.00$ & $9: 05: 06.5$ & - & $18.56 \pm 0.03$ & $16.36 \pm 0.04$ & - & 2.20 & 20 \\
\hline
\end{tabular}

Notes: names are from De Pree et al. (1997). Sources associated with RQ, S, and W49 South are likely to be multiple unresolved objects; nebular emission near these sources complicates the determination of the background and likely makes the errors in the photometry larger than the quoted formal photometric errors. Masses are inferred from the extinction-corrected $K$ magnitudes, a distance of $11.4 \mathrm{kpc}$, and $4 \times 10^{5} \mathrm{yr}$ stellar tracks from Lejeune \& Schaerer (2002) with solar metallicity and enhanced mass loss. Objects with only $H \& K$ s magnitudes may have an unknown $K$ s excess which could severely affect the inferred mass. Five objects have inferred masses above $120 M_{\odot}$, which is likely due to a $K$ s excess and/or multiplicity. Errors of $10-20 \%$ in the mass estimate are expected simply from the uncertainty in the distance.

regions will appear to be the "core" of the star cluster. The projected geometry of the region is highly suggestive of triggering; the "ring" of UC H II regions is at the border of the ionized bubble surrounding Cluster 1.

What does the core of Cluster 1 hold? Given the high internal extinction, we are likely to be incomplete in our nearinfrared census of star formation and therefore a total mass or density estimate. The core is crowded; high spatial resolution observations are needed to accurately determine the stellar density in the core. Taken at face value and without correcting for the large extinction, the cluster core appears to be significantly less dense than the Arches cluster, NGC 3603, or 30 Doradus. If it is truly less dense, then the different formation environment of W49A, at a Galactocentric distance of $8 \mathrm{kpc}$, may be an important clue for understanding the processes which drive clustered star formation.

The subclustering phenomenon is useful to describe the star formation pattern in the W49A molecular cloud. When the cloud has ceased forming stars, the resulting stellar group will likely be called a "cluster". At the time of current observation, the massive star formation does not appear to be distributed uniformly throughout the region, or with a radial dependence relative to a cluster "center". Rather it is better described as occurring in "subclusters". In this sense we could count
4-5 subclusters within $\sim 13$ pc using the combined NIR and radio observations: Cluster 1, the (Welch) "ring" of UC H II regions, W49A South, the RQ complex, and perhaps the CC source. We speculate that star formation within a subcluster is essentially synchronized, and a massive star cluster is a collective of several (or many) subclusters.

\subsection{Total stellar mass}

We can make an estimate for the total stellar mass of the W49A star cluster by counting stars with masses greater than $20 M_{\odot}$ and using a Salpeter slope for the mass function. We take upper and lower mass limits as $120 M_{\odot}$ and $1 M_{\odot}$, respectively. For Cluster 1, we find 54 stars within 45", implying a total mass of $\sim 1 \times 10^{4} M_{\odot}$. In our entire field, we count 269 stars with masses $\geq 20 M_{\odot}$, implying a total mass of $5-7 \times 10^{4} M_{\odot}$. The stars we have identified as massive stars are certainly contaminated by background objects, but we are also certainly incomplete in our census due to extinction and angular resolution. The extent to which these effects cancel each other (or not) is unknown. Even if the stellar mass estimate for W49A is a factor of 2 too high, W49A is as massive or more massive than any known young Galactic star cluster. We also note that it is possible, perhaps even likely, that we have not yet detected 
the most massive young star clusters in the Milky Way (e.g. Hanson 2003).

It is important to note that this is a lower limit to the final stellar mass, as there is circumstantial and direct evidence for ongoing star formation in this region. There is abundant molecular gas, and hot cores near the ring of UC H II regions (Wilner et al. 2001; McGrath et al. 2004). The densest region of the molecular cloud is north of the "ring" of UC H II regions, which is completely extincted even in our $K$ s image. This is the most likely place for massive stars in earlier stages of formation than probed with existing observations. What we observe in $\mathrm{W} 49 \mathrm{~A}$ is a region with massive stars at various evolutionary stages, from hot cores to UC H II regions to naked OB stars, similar to W43 (Blum et al. 1999; Motte et al. 2003) and the significantly less massive W75N (Shepherd et al. 2003).

\section{Conclusions}

We have presented a more comprehensive investigation into our previous discovery of stellar clusters in the giant radio HII region W49A (Alves \& Homeier 2003). Our observations clearly show a massive star cluster adjacent to the UC H II regions ( 2 pc distant). This means that the W49A region began forming stars earlier than previously thought, and that the UC HII regions which have long been known to radio astronomers are not the first generation of massive stars. We use these data to estimate a total stellar mass in this region of 5-7 $\times 10^{4} M_{\odot}$, and a total mass for Cluster 1 of $1 \times 10^{4} M_{\odot}$. Since molecular gas is abundant, this is a lower limit to the final stellar mass of the cluster.

With these observations, W49A joins the list of Galactic giant radio H II regions where two or more phases of massive star formation coexist. This means that the formation of massive stars is not completely synchronized, but that there is some spread in age. The magnitude of the spread could be investigated with spectra of the relatively unembedded massive stars and the lifetime of UC HII regions, although this lifetime is only poorly known. With the current observations and the presence of dense molecular gas in the central few pc, a reasonable guess for the age spread is $1 \mathrm{Myr}$.

The last point we would like to make is that the subclustering phenomenon is essential for the description of star formation in the W49A molecular cloud, at least as traced by the massive stars. However, there also exists evidence for subclustering in lower-mass star-forming regions (Lada et al. 1996; Testi et al. 2000), which is reproduced in star formation simulations (Bonnell et al. 2003). Possible examples of subclustering in extragalactic star clusters are: SSC-A in NGC 1569 and NGC 604 in M 33. SSC-A in NGC 1569 has a stellar concentration with red supergiants and another with Wolf-Rayet stars (Gonzalez Delgado et al. 1997; de Marchi et al. 1997; Hunter et al. 2000; Origlia et al. 2001). The massive stars in NGC 604 are subclustered, but the region itself is of sufficiently low density to be termed a Scaled OB Association (SOBA) rather than a star cluster (Maíz-Apellániz 2001). The applicability of the subclustering description to other young massive Galactic star clusters remains to be seen, but we conclude that it is a useful concept for describing and understanding massive star formation in the W49 GMC.

Acknowledgements. We would like to thank the anonymous referee for careful readings and comments which improved the paper. N.H. acknowledges and thanks the European Southern Observatory (ESO) Studentship Programme which provided support during the early stages of this work. We are pleased to acknowledge Miguel Moreira for discussions and assistance with the observations, Robert Simon for providing molecular line data on W49's giant molecular cloud, where the clusters are embedded, and Chris De Pree for providing radio continuum data of the H II regions associated with W49A.

\section{References}

Alves, J., \& Homeier, N. 2003, ApJ, 589, L45

Barbosa, C. L., Damineli, A., Blum, R. D., \& Conti, P. S. 2003, AJ, 126, 2411

Bessell, M. S., \& Brett, J. M. 1988, PASP, 100, 1134

Blum, R. D., Damineli, A., \& Conti, P. S. 1999, AJ, 117, 1392

Blum, R. D., Conti, P. S., \& Damineli, A. 2000, AJ, 119, 1860

Blum, R. D., Damineli, A., \& Conti, P. S. 2001, AJ, 121, 3149

Blum, R. D., Schaerer, D., Pasquali, A., et al. 2001, AJ, 122, 1875

Bonnell, I. A., Bate, M. R., \& Vine, S. G. 2003, MNRAS, 343, 413

Brandl, B., Brander, W., Eisenhauer, F., et al. 1999, A\&A, 352, 69

Brandner, W., Grebel, E. K., Chu, Y.-H., et al. 2000, AJ, 119, 292

Brogan, C. L., \& Troland, T. H. 2001, ApJ, 550, 799

Cardelli, J. A., Clayton, G. C., \& Mathis, J. S. 1989, ApJ, 345, 245

Churchwell, E. 2002, ARA\&A, 40, 27

Clark, J. S., \& Negueruela, I. 2002, A\&A, 396, L25

Comerón, F., Pasquali, A., Rodighiero, G., et al. 2002, A\&A, 389, 874

Conti, P. S., \& Blum, R. D. 2002, ApJ, 564, 827

de Marchi, G., Clampin, M., Greggio, L., et al. 1997, ApJ, 479, L27

De Pree, C. G., Mehringer, D. M., \& Goss, W. M. 1997, ApJ, 482, 307

De Pree, C. G., Wilner, D. J., Goss, W. M., Welch, W. J., \& McGrath, E. 2000, ApJ, 540, 308

Drissen, L., Moffat, A. F. J., Walborn, N. R., \& Shara, M. M. 1995 , AJ, 110, 2235

Eisenhauer, F., Quillenbach, A., Zinnecker, H., \& Genzel, R. 1998, ApJ, 498, 278

Figer, D. F., Najarro, F., Gilmore, D., et al. 2002, ApJ, 581, 258

Figuerêdo, E., Blum, R. D., Damineli, A., \& Conti, P. S. 2002, AJ, 124,2739

Garay, G., \& Lizano, S. 1999, PASP, 111, 1049

Gonzalez Delgado, R. M., Leitherer, C., Heckman, T., \& Cerviño, M. 1997, ApJ, 483, 705

Gwinn, C. R., Moran, J. M., \& Reid, M. J. 1992, ApJ, 393, 149

Hanson, M. M. 2003, ApJ, 597, 957

Homeier, N. L., Blum, R. D., Conti, P. S., \& Damineli, A. 2003, A\&A, 397,585

Hunter, D. A., O’Connell, R. W., Gallagher, J. S., \& Smecker-Hane, T. A. $2000, A J, 120,2383$

Johnson, K. E., Kobulnicky, H. A., Massey, P., \& Conti, P. S. 2001, ApJ, 559, 864

Johnson, K. E., Indebetouw, R., \& Pisano, D. J. 2003, AJ, 126, 101

Johnson, K. E., \& Kobulnicky, H. A. 2003, ApJ, 597, 923

Kent, S. M., Dame, T. M., \& Fazio, G. 1991, ApJ, 378, 131

Kim, K., \& Koo, B. 2002, ApJ, 575, 327

Knödlseder, J. 2000, A\&A, 360, 539

Lada, C. J., Alves, J., \& Lada, E. A. 1996, AJ, 111, 1964

Lejeune, T., \& Schaerer, D. 2001, A\&A, 366, 538

Maíz-Apellániz, J. 2001, ApJ, 563, 151

Massey, P., \& Hunter, D. A. 1998, ApJ, 493, 180 
McGrath, E. J., Goss, W. M., \& De Pree, C. G. 2004 ApJS, in press Mezger, P. G., Schraml, J., \& Terzian, Y. 1967, ApJ, 150, 807

Moffat, A. F. J., Drissen, L., \& Shara, M. M. 1994, ApJ, 436, 183

Moffat, A. F. J., et al. 2002, ApJ, 127, 1014

Moorwood, A., Cuby, J. G., \& Lidman, C. 1998, The Messenger, 91, 9

Motte, F., Schilke, P., \& Lis, D. C. 2003, ApJ, 582, 277

Nürnberger, D. E. A., \& Petr-Gotzens, M. A. 2002, A\&A, 382, 537

Nürnberger, D. E. A., Bronfman, L., Yorke, H. W., \& Zinnecker, H. 2002, A\&A, 394, 253

Origlia, L., Leitherer, C., Aloisi, A., Greggio, L., \& Tosi, M. 2001, AJ, 122,815

Persson, S. E., Murphy, D. C., Krzeminski, W., Roth, M., \& Rieke, M. J. 1998, AJ, 116, 247

Rathborne, J. M., Burton, M. G., Brooks, K. J., et al. 2002, MNRAS, 331,85

Rieke, G. H., \& Lebofsky, M. J. 1985, ApJ, 288, 618

Serabyn, E., Shupe, D., \& Figer, D. F. 1998, Nature, 394, 448

Shaver, P. A., \& Goss, W. M. 1970, AuJPA, 14, 113

Shepherd, D. S., Testi, L., \& Stark, D. P. 2003, ApJ, 584, 882

Simon, R., Jackson, J. M., Clemens, D. P., Bania, T. M., \& Heyer, M. H. 2001, ApJ, 551, 747

Smith, L. F., Mezger, P. G., \& Biermann, P. 1978, A\&A, 66, 65
Smith, N., Jackson, J. M., Kraemer, K. E., et al. 2000, ApJ, 540, 316

Sparke, L. S., \& Gallagher, J. S. 2000, Galaxies in the universe: an introduction, ed. L. S. Sparke, \& J. S. Gallagher (Cambridge, UK: Cambridge University Press)

Sternberg, A., Hoffmann, T. L., \& Pauldrach, A. W. A. 2004, ApJ, in press

Stolte, A., Grebel, E. K., Brandner, W., \& Figer, D. F. 2002, A\&A, 394, 459

Stolte, A., Brandner, W., Brandl, B., Zinnecker, H., \& Grebel, E. 2004, AJ, 128, 765

Sung, H., \& Bessell, M. S. 2004, AJ, 127, 1014

Testi, L., Sargent, A. I., Olmi, L., \& Onello, J. S. 2000, ApJ, 540, L53

Vacca, W. D., Johnson, K. E., \& Conti, P. S. 2002, AJ, 123, 772

Walborn, N. R. 1995, Rev. Mex. Astron. Astrofis. Conf. Ser., 2, 51

Walborn, N. R., Barbá, R. H., Brandner, W., et al. 1999, AJ, 117, 225

Walborn, N. R., Maíz-Apellániz, J., \& Barbá, R. H. 2002, AJ, 124, 1601

Walmsley, M. 1995, Rev. Mex. Astron. Astrofis. Conf. Ser., 1, 137

Welch, W. J., Dreher, J. W., Jackson, J. M., Terebey, S., \& Vogel, S. N. 1987, Science, 238, 1550

Wilner, D. J., De Pree, C. G., Welch, W. J., \& Goss, W. M. 2001, ApJ, 550, L81 